\title{
SECURITY THROUGH INTEGRATION: TOWARDS A COMPREHENSIVE FRAMEWORK FOR DEVELOPMENT AND CERTIFICATION
}

\author{
Bertrand Marquet, Adrian Rossi, Francois J.N. Cosquer \\ Alcatel Research and Innovation Security Group \\ \{Bertrand.Marquet, Adrian.Rossi, Francois.Cosquer\}@alcatel.com
}

\begin{abstract}
Today secured infrastructures are more and more being built by integration of both in-house components and policies, and off-the-shelf products. This poses several difficulties for developers. Despite, or because, the numerous choice of security products, it is difficult to build, in a coherent way, a comprehensive set of security features within distributed applications without severely increasing complexity and decreasing scalability of globally secured applications. Another difficulty appears when security certification needs to be achieved for such an integrated security architecture. In this paper we propose an alternative approach to building secured applications through integration which will additionally guarantee a means to achieve security certification, such as ISO 15408 (Common Criteria) [4].
\end{abstract}

Keywords: security, development, integration, certification, common criteria

\section{THE NEED FOR A GENERIC INTEGRATION LAYER}

As telecommunication infrastructures became more and more complex, the development of the associated software and protocols is more and more realized by integration. This is particularly true when securization has to be considered. Developers are being challenged to make interoperate integrated technologies in order to satisfy strong security requirements and assess the level of security of such infrastructures.

As a consequence, a security integration infrastructure should be defined as a generic security layer comprised of middleware technologies [3] that provide communication, integration, organization, and coordination of security services. 
A considerable number of studies exist discussing how one should provide a generic security layer $[1,7,8,10,11]$ some using Common Criteria as a guideline [6] or using organizational requirements as a model $[5,9]$. As an example RFC-2078 defined the Generic Security Service API (GSS-API). It was originally conceived to provide portability between distributed security architectures based on a security scheme such as Kerberos [13]. GSS-API is based on two pieces: a generic interface to the set of security services and a collection of underlying mechanisms providing the security services. GSSAPI still remains difficult for non-security aware developers and has lost favor mainly due to complexity and scalability.

In our approach, we wish to provide a generic integration layer accessible through a generic API but also we want to achieve a trade-off between genericity and complexity [1]. We believe the success of such a generic API should be measured by how easily it can be understood by non security experts. The concrete realization of this approach is called the Generic Trusted Computing base (G-TCB).

\section{GENERIC TRUSTED COMPUTING BASE CONCEPT}

The classical process is to create or integrate the required mechanisms into the business application code in order to fulfill a determined list of security requirements. This approach has well-known limitations; it is difficult to:

- control the global behavior of the resulting secured architecture;

- add, replace, retrieve or maintain security functions;

- provide evidence, traceability to achieve and maintain security certification.

Introducing the G-TCB concept, we have proposed an alternative approach using an intermediate set of components that steer the integration of security mechanisms. This intermediate set of components should be able to fulfill a comprehensive set of high-level security requirements in order to address both existing and as yet unknown security needs. As a consequence, our approach is based on the assumption that security code and business code will be completely separated.

Today, telecommunication applications represent several tens of millions of lines of code, and consequently they can not be completely bug-free. The forced independence of the business layer from the security layer guarantees at least that any software failure will have a minimal repercussion on the security of the associated business applications.

This strong separation also allows the global definition, in terms of a security state machine, of the G-TCB components. This would be difficult, even close 
to impossible, to do for current business software in which security code is deeply embedded within the business logic. As a result, the global security behavior of a distributed application is realized by collaborations between the G-TCB components.

\subsection{DEFINITION OF INTEGRATION COMPONENTS}

The G-TCB is defined using common practices for secured development, for example:

- by using programmaticaly safe languages with strong security features such as Java,

- by implementing most security architectural patterns [12],

- by applying basic security principles such as least privileges, etc.

The main focus of our research effort to date has been to define the nature of the components which make up our generic security integration framework. We determined that these components must satisfy the following objectives. They have to:

- be specified at a high enough level to be able to provide a generic view of the underlying security, i.e an understandable view for business developers;

- represent a coherent and comprehensive set of components able to address at least the majority of security requirements that might arise;

- be sufficiently configurable in their implementation that one is able to integrate most security mechanisms or COTS, only at the time of deployment and only through a configuration interface (i.e. without the need to write any additional source code).

Several methodologies, standards and frameworks have been evaluated in order to provide us with a path to achieving the above objectives. We finally decided [2] to base our framework on the newly defined standard for specifying and evaluating the security of an IT product, i.e. the ISO 15408 standard, also referred to as the Common Criteria.

The Common Criteria incorporates a common set of constructs and a language in which to express and communicate the relevant aspects of IT Security, and permits those responsible for IT security to benefit from the prior experience and expertise of others. Common Criteria represent the culmination in terms of effort for achieving a comprehensive and coherent catalog of IT Security requirements, both functional and assurance related.

Our approach provides an innovative use of Common Criteria standard. 


\subsection{CHARACTERIZATION}

The interface and the behaviour of G-TCB component has been fully described using the Common Criteria Part 2 "Functional requirements" components taken at a Protection Profile level i.e. at a generic level. Each requirements class or family has been considered, as the description of one or several integration components (i.e small enough to be able to formally describe its behaviour with a finite state machine).

The characterization has been completed by performing equivalent operations in the software component than in the Common Criteria component. This is achieved by multi-instantiation, parameterization and configuration at application deployment time.

The integration layer is composed of about twenty integration components directly derived from the Common Criteria functional requirements class and family. This layer also needs some intermediate objects to represent entities and security attributes manipulated (and only) by the integration components.

\subsection{THE LOW LEVEL INTERFACE}

In order to provide generic integration components, the integration layer makes use of several existing technologies such as Java Security and CorbaSec frameworks which provide levels of integration but not in a unified or consistent way; this is where our approach will be proved useful by combining and abstracting some or parts of these frameworks as a low level interface. As an example, the Java Authentication and Authorization Service (JAAS)[15] has been selected to provide the low-level authentication interface. It has been used directly to implement authentication mechanisms through modules or indirectly to provide interface adaptors or authentication protocols adaptors in the form of clients.

\section{PROTOTYPING}

A first prototype using four G-TCB components has been built to provide authentication, data protection and audit for a sample distributed application.

\section{IMPROVING THE CERTIFICATION PROCESS}

The concepts and architecture described above also increase the effectiveness and reduce delays in achieving security assurance certification.

In addition to the increased level of assurance by construction considerations, usage of integration components that have been directly derived, defined 
and implemented from the common criteria, improve the assurance process evaluation by:

- facilitating the traceability, the identification and production of evidence of suitability of the integrated security functions;

- reducing the cost of the evaluation of a composed architecture by integration of already certified products;

- simplifying the maintenance of the certified architecture by limitation on the impact of changed security functions.

Those advantages may represent subsequent cost savings for a product under certification maintenance with frequent evolutions, especially for low or intermediate security assurance level (i.e. up to EAL 3).

\section{FUTURE PROMISE}

The technique described in this paper holds great promise for providing an extended framework for securing applications by integrating in house components as well as third party products. Tools such as NIAP [14] Common Criteria tools are also under investigation as a means of completing this approach; we will then be able to begin at the specification of the requirements and move through the whole software lifecycle, leading to the certification of the resulting integrated solutions.

\section{CONCLUSION}

We have shown in this paper that the security of distributed systems provided through integration is a problem that telecommunications developers have to face today. Without any help, methodology or framework such as the one we have defined in this paper, it becomes an extremely uncertain and challenging task. Hence, providing developers with necessary tools and support so that they are able to address the problem in an efficient manner, becomes a priority.

\section{References}

[1] D. Gollmann. Computer Security, John-Wiley, 1999.

[2] B. Marquet, and C. Gustave. Common Criteria, a Foundation for a Comprehensive Security Framework. Proceedings of the 3rd International Conference on Common Criteria, Ottawa, Canada, May 2002.

[3] Aberdeen Group. E-Business Infrastructure Integration: Practical Approaches. White paper, November 2001. 
[4] International standard ISO/IEC 15408. Common Criteria for Information Technology Security Evaluation (parts 1-3), version 2.1. CCIB-98-026, August 1999.

[5] J. Leiwo, C. Gannage, and Y. Zheng. Organizational Modeling for Efficient Specification of Information Security Requirements. In Advances in Databases and Information And Systems, Proceedings of the 3rd EastEuropean Conference. Lecture Notes in Computer Science, Maribor, Slovenia, September 1999, Springer-Verlag.

[6] K.Md. Khan, J.Han, and Y. Zheng. Characterizing User Data Protection of Software Components. In the Proceedings of the 2000 Australian Software Engineering Conference.

[7] C. Irvine, and T. Levin. A Note on Mapping User-Oriented Security Policies to complex Mechanisms and Services. Technical report NPS-CS-9908, Naval postgraduate School, Montray, CA, June 1999.

[8] R. Spencer, S. Smalley, P. Loscocco, M. Hibler, D. Andersen, and J. Lepreau. The Flask Security architecture: System Support for diverse Security Policies. Technical report UUCS-98-014, University of Utah, US, August 1998.

[9] J. Leiwo, C. Garnage, and Y. Zheng. Harmonizer, A Tool for Processing Information Security Requirements in Organizations. In the Proceedings of the Third Nordic Workshop on Secure IT Systems (NORDSEC98), Trondheim, Norway, November 1998.

[10] J. Leiwo, C. Hale, P. Homburg, C. Garnage and A.S. Tanenbaum. A Security Design for a Wide-Area Distributed System. Pre-proceedings of the second International Conference on Information security and Cryptology (ICISC99), pages 105-116, Seoul, Korea, December 1999.

[11] A. Weber. Benefits of a Universal Security Framework. To appear in World Market Research Centre (ed.) Business Briefing: electronic Commerce, London 2000.

[12] J. Yoder, and J. Barcalow. Architectural Patterns for Enabling Application Security. In Proceedings of the 4th Pattern Languages of Programming, Monticello, Il. September 1997.

[13] RFC1510 - The Kerberos Network Authentication Service (V5).

[14] Common Criteria Tools. Available at http://niap.nist.gov/cctools.

[15] C. Lai, L. Gong, L. Koved, A. Nadalinm and R. Schemers. Java Authentication and Authorizations in the Java Platform. 19-th Annual Computer Security Applications Conference, Phoenix, AZ, December 1999. 